\title{
Symmetry and asymmetry during magnetization reversal in exchange biased multilayers and bilayers
}

\author{
Amitesh Paul,* Emmanuel Kentzinger, Ulrich Rücker, and Thomas Brückel \\ Institut für Festkörperforschung, Forschungszentrum Jülich GmbH, D-52425 Jülich, Germany
}

(Received 8 December 2005; published 20 March 2006)

\begin{abstract}
We have studied the magnetization reversal process in continuous: $[\mathrm{Co} / \mathrm{CoO}]_{20}$ and separated: $[\mathrm{Co} / \mathrm{CoO} / \mathrm{Au}]_{20}$ exchange-biased polycrystalline multilayers (MLs). For continuous ML, reversal proceeds sequentially starting with the bottom (top) Co layer for increasing (decreasing) field. Each Co layer remagnetizes symmetrically for both field branches in a nonuniform mode similarly as we have observed earlier for $[\mathrm{IrMn} / \mathrm{CoFe}]_{3 / 10}$ MLs [Phys. Rev. B. 70, 224410 (2004)]. By polarized neutron reflectivity, we observe increasing exchange bias field strengths down the stack. However, usual asymmetric reversal is observed for the separated ML. We explain the different magnetization behavior within a simple and general model. The increased anisotropy energy for continuous ML is responsible for the nonuniform symmetric reversal as the angular dependencies for reversal are guided by the relative strengths of exchange, anisotropy, and Zeeman energies.
\end{abstract}

DOI: 10.1103/PhysRevB.73.092410

PACS number(s): 75.70.Cn, 61.12.Ha, 75.60.Jk

Direct exchange coupling between the ferromagnet (FM) and antiferromagnet $(\mathrm{AF})$ layers give rise to a unidirectional magnetic anisotropy called exchange bias $H_{E B}$ (Ref. 1) when a ferromagnet in contact with an antiferromagnet is cooled below the blocking temperature of the AF in an external field $H_{F C}$.

Asymmetric hysteresis loops due to a different magnetization reversal process in different branches of the hysteresis loop are common ${ }^{2-6}$ in exchange biased systems. Neutron scattering under grazing incidence with polarization analysis has been proven decisive for identification of the reversal mechanism. Two mechanisms can be distinguished: uniform magnetization reversal by magnetization rotation ${ }^{3-6}$ and nonuniform magnetization reversal by domain nucleation and growth. Magnetization rotation is identified by a significant increase of the specular reflectivities in the spin-flip (SF) channels $\left(R_{+-}\right.$and $\left.R_{-+}\right)$, which correspond to in-plane magnetization components perpendicular to the guiding field $H_{a}$ applied collinear to $H_{F C}$. Reversal by domain nucleation and propagation (nonuniform magnetization reversal) does not provide enhanced SF intensities because the magnetization is always collinear to $H_{a}$. Reversal mechanisms are observed for the $\mathrm{Co} / \mathrm{CoO}$ bilayer systems, ${ }^{5,6}$ where the domain wall motion occurs for the decreasing (positive to negative) and magnetization rotation for the increasing (negative to positive) field sweeping direction of $H_{a}$ for the hysteresis loop with respect to negative direction of $H_{F C}$. This behavior is just opposite to that reported in Ref. 4. Theoretically the interpretation of the magnetization reversal was discussed in Ref. 7 where it was shown that depending on $\theta$, the angle between $H_{F C}$ and the AF anisotropy axis, the reversal mode is either by coherent rotation for both loop branches or asymmetric with a nonuniform reversal for the decreasing branch.

Very recently, Paul et al. ${ }^{8}$ have shown symmetric and sequential reversal for polycrystalline $\left[\mathrm{Ir}_{20} \mathrm{Mn}_{80}(6.0 \mathrm{~nm}) / \mathrm{Co}_{80} \mathrm{Fe}_{20}(3.0 \mathrm{~nm})\right]_{10}$ multilayers. Here sequential refers to a process, where different layers reverse their magnetization at different field strengths one after an- other. This reversal mode-symmetric, and nonuniformcorresponds to the situation $\theta=0$, considered unlikely to occur in experiments. ${ }^{7}$ Interestingly, however, the samples were multilayers (MLs) unlike the bilayer specimens investigated experimentally $^{4-6}$ as well as theoretically. ${ }^{7}$ A ML differs from a bilayer in the sense that the FM interfaces are pinned by the AF layer on both sides (AF-FM-AF), whereas a bilayer (FM-AF) has only one such interface.

In the present Brief Report, we investigate the layer-bylayer evolution of the magnetization configuration of polycrystalline $\mathrm{CoO} / \mathrm{Co}$ multilayers (MLs) along a full magnetization loop by specular and off-specular polarized neutron reflectometry (PNR). The field cooling axis and the reflectometer axis were the same, thus ensuring the $\theta=0$ situation for all samples. We use two sets of MLs, one is a continuous repetition of $\mathrm{CoO}-\mathrm{Co}$ interfaces $[\mathrm{CoO}(7.0 \mathrm{~nm}) / \mathrm{Co}(11.0 \mathrm{~nm})]_{20} / \mathrm{Au}(50.0 \mathrm{~nm})$ labeled as ML-C and another with separate interfaces $[\mathrm{CoO}(8.0 \mathrm{~nm}) / \mathrm{Co}(15.0 \mathrm{~nm}) / \mathrm{Au}(46.0 \mathrm{~nm})]_{20}$, separated by $\mathrm{Au}$ at each bilayer interface and labeled as ML-S. Similar separate MLs have been also investigated earlier. ${ }^{5,6}$ Here, we particularly study the ML-C and compare it with the ML-S. We find a sequential switching of the layers that we relate to the microstructural evolution along the stack for the ML-C. The reversal proceeds for both loop branches in a nonuniform mode for the continuous case whereas for the separate case, we observe the nonuniform and uniform mode of reversal depending upon the branch of the field cycle.

The exchange-biased systems are structures of $\mathrm{CoO} / \mathrm{Co}$ prepared on oxidized $\mathrm{Si}$ substrates in a dc magnetron sputtering system with a base pressure of $1 \times 10^{-7}$ mbar. The deposition starts by growing a Co layer, which is subsequently oxidized in situ to obtain the antiferromagnetic $\mathrm{CoO}$ layer. ${ }^{9}$ We verified all layer thicknesses by fitting specular $\mathrm{X}$-ray reflectivity spectra using Parratt's formalism. ${ }^{10}$ Magnetization loops are measured by means of a superconducting quantum interference device (SQUID) at $10 \mathrm{~K}$ after field cooling in an external field of $\pm 5 \mathrm{kOe}$ from room tempera- 


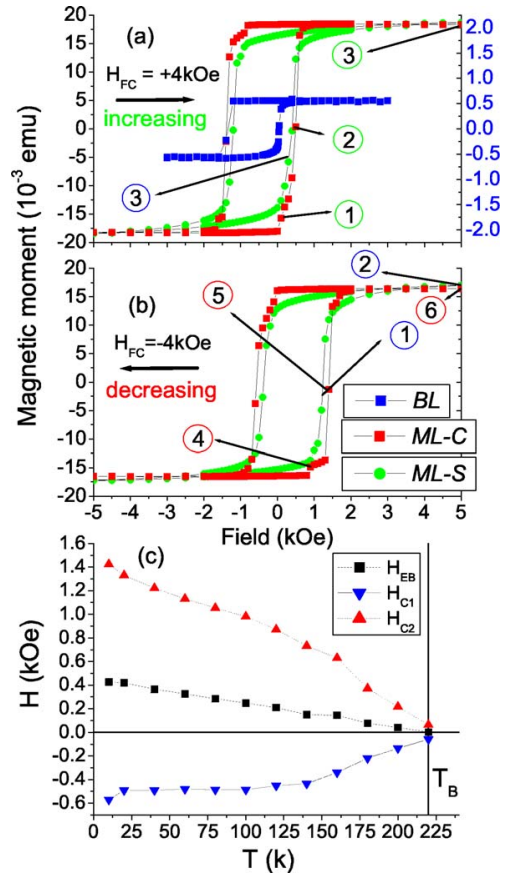

FIG. 1. (Color online) SQUID hysteresis loops at $10 \mathrm{~K}$ for the continuous: ML-C (red) and separate: ML-S (green) MLs when field cooled at (a) $+4.0 \mathrm{kOe}$ and (b) $-4.0 \mathrm{kOe}$. The bilayer sample: BL-S (blue) in (a) is also shown for comparison. The $y$ axis for the BL-S is labeled in blue. (c) Temperature dependence of the coercivities $H_{C 1}$ (negative branch), $H_{C 2}$ (positive branch), and the loop shift $H_{E B}$ for the ML-C when $H_{F C}=-4.0 \mathrm{kOe}$. The numbers in circles are the field values for which we show the PNR spectra.

ture to $10 \mathrm{~K}$, i.e., well below the blocking temperature $T_{B}$ $\approx 220 \mathrm{~K}$ of $\mathrm{CoO}$.

We performed the PNR measurements at the polarized reflectometer HADAS (Ref. 11) with polarization analysis at the Jülich research reactor FRJ-2 (DIDO). The instrumental conditions were the same as discussed earlier in Ref. 8. We use a continuous flow He-cryostat equipped with a temperature controller for cooling the specimens down to $10 \mathrm{~K}$.

Figures 1(a) and 1(b) show SQUID magnetization loops for the specimens cooled down at $H_{F C}=+4.0 \mathrm{kOe}$ and $-4.0 \mathrm{kOe}$, respectively, for both the ML-C and ML-S. We also show the loop for a bilayer (BL-S) of composition $[\mathrm{Co}(11.0 \mathrm{~nm}) / \mathrm{CoO}(7.0 \mathrm{~nm})]_{1}$ for comparison. The bilayer shows an usual asymmetric hysteresis loop: a sharp jump of magnetization for the decreasing (nonuniform reversal) and gradual variation (uniform reversal) for the increasing branch. By applying $+/-H_{F C}$, we make the samples undergo the magnetization reversal for increasing/decreasing field branches of the hysteresis loop by applying only positive fields. This is to avoid any depolarization of the beam during neutron measurements. From the respective coercive fields $H_{C 1}$ (for negative branch) and $H_{C 2}$ (for positive branch), we find a large increase ( $\sim 500 \mathrm{Oe})$ in $H_{C 2}$ in MLs compared to the BL-S sample. Training effects are seen for both sets of specimens up to the second field cycle. We always use virgin samples for the neutron measurements and then also measure along the second cycle, thereby investigating the reversal process with and without training effects. The $T_{B}$ is determined for our samples at $220 \mathrm{~K}$ as shown in Fig. 1(c). To avoid any training effects we heat up the sample above the Néel temperature of $\mathrm{CoO}(293 \mathrm{~K})$ each time before measuring at a particular temperature. The $H_{E B}$ at $10 \mathrm{~K}$ for the ML-S is 414 Oe (ML-S)-lower than that of BL-S where it is $650 \mathrm{Oe}$. The $H_{E B}$ for the ML-C shows a variation with increasing number of bilayers. The atomic force microscopy (not shown) of the top Au layer (for ML-C and ML-S) reveals a grain size of $\approx 200 \mathrm{~nm}$ compared to $50 \mathrm{~nm}$ for BL-S. Smaller grains are responsible for a larger number of uncompensated spins and thereby increased $H_{E B}$ values. Thus the presence of the separating $50.0 \mathrm{~nm}$ thick Au layer on top of the first bilayer for the ML-S sample is thought to have increased its grain sizes.

All four polarization channels of the specular reflectivity are measured at 15 different external fields $H_{a}$, six of which

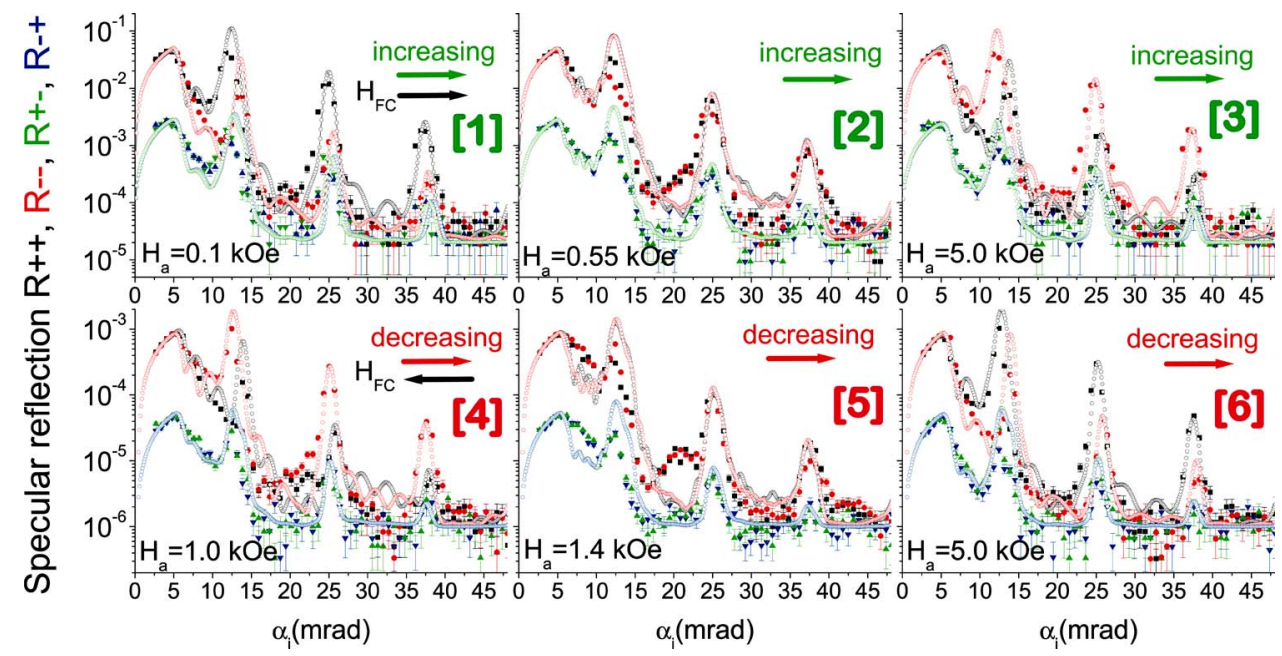

FIG. 2. (Color online) Measured (closed symbols) and fitted (open circle) NSF $\left(R_{++}\right.$and $\left.R_{--}\right)$and SF $\left(R_{+-}\right.$and $\left.R_{-+}\right)$reflectivity patterns of continuous: $\mathrm{SiO}_{2} /[\mathrm{Co}(11.0 \mathrm{~nm}) / \mathrm{CoO}(7.0 \mathrm{~nm})]_{\times 20} / \mathrm{Au}(50.0 \mathrm{~nm}) \mathrm{ML}$ at different applied fields $H_{a}$ along increasing/decreasing branches of the hysteresis loops, where $H_{F C}=+4.0 /-4.0 \mathrm{kOe}$, respectively. The numbers correspond to the points of the hysteresis loop labeled in Fig. 1. 


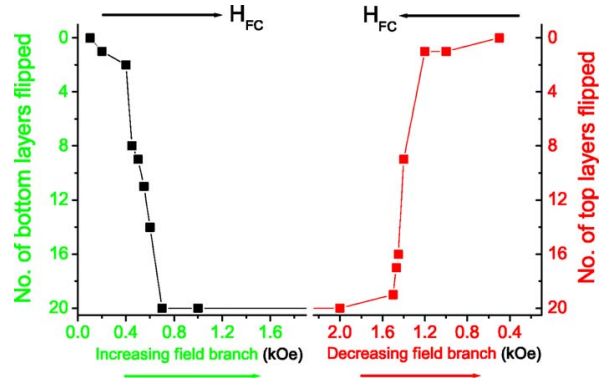

FIG. 3. (Color online) Switching sequence of the ML-C as obtained from the fits.

are shown in Fig. 2 (circled numbers in Fig. 1) together with least-squares fits based on an extension of the Paratt formalism ${ }^{10}$ to magnetic MLs. ${ }^{12}$ The three peaks of the ML-C in the NSF channels $\left(R_{++}\right.$and $\left.R_{--}\right)$are the first, second, and third order Bragg reflections of the ML. The corresponding weak peaks in the SF channels $\left(R_{+-}\right.$and $\left.R_{-+}\right)$can be reproduced in the fits by taking into account the polarization inefficiencies of our setup (therefore the intensities are not due to SF processes). $R_{++}$and $R_{--}$are almost equal at $H_{a}=550 \mathrm{Oe}$ [2] on the increasing and at $H_{a}=1400$ Oe [5] on the decreasing branch and signify that the reversal for both loop branches proceeds via a state with an almost vanishing magnetization component collinear to $H_{a}$. We think that some enhanced scattering intensities at the reversal points are most likely due to small fluctuation of the magnetization about their mean direction along the field as they have been seen to almost disappear in the trained state. ${ }^{13}$ For all other fields $R_{++}$or $R_{--}$dominates and reflects a net magnetization collinear with $H_{a}$, while the SF intensities are always much weaker. The presence of off-specular intensity (not shown) for the NSF channels in the Bragg sheets at higher fields [3] is related to the vertical correlation of the interface roughness in the ML. We do not observe any grain size induced small length scale magnetic fluctuations as has been observed for IrMn/CoFe MLs. ${ }^{8}$

We fit the specular intensities as described earlier in Ref. 8 by only considering deviations from the purely collinear, single domain configurations (i.e., $\theta_{\mathrm{i}}=0$ or $180^{\circ}$ ). Co layers $i=1, \ldots, 20$ are described by the mean magnetization amplitude $\left\langle M_{\mathrm{i}}\right\rangle$ and the mean angular deviation from the collinear alignment $\left\langle\Delta \theta_{\mathrm{i}}\right\rangle$. The fitted $\left\langle M_{\mathrm{i}}\right\rangle$ does not show significant variations. The results are shown in Fig. 3 for ML-C: de- creasing $H_{a}$ switches the Co layers sequentially from the top to the bottom, and in increasing $H_{a}$ the reversal proceeds in the opposite direction. This sequential switching of the layers is similar but opposite to that reported in Ref. 8. We explain this by a different structural evolution from bottom to top within the ML. This also indicates that the Co layers are magnetically uncoupled in the stack. In contrast to Ref. 8 we do not directly observe the variation of grain sizes with increasing layers for the present case, as our samples are capped with a Au layer. However, we observe bigger grains from the top $\mathrm{Au}$ layer than that of the bilayer. Sequential reversal of the layers indicates a decreasing strength of the exchange coupling with number of layers. This symmetric magnetization reversal process, without coherent rotation, remains the same even for the second field cycle, i.e., in the trained state. ${ }^{13}$

For the ML-S, reflectivity curves along with their fits are shown in Fig. 4. $R_{++}$and $R_{--}$are mostly similar at $H_{a}$ $=1100 \mathrm{Oe}$ [1] on the decreasing and at $H_{a}=200 \mathrm{Oe}$ [3] on the increasing branch signifying magnetization reversal. We also show the saturation state at $H_{a}=5.0 \mathrm{kOe}$ [2]. We find that a reduced Co magnetization in the decreasing branch and the SF signal is significant only for the increasing field branch. This observation can be ascribed to asymmetric reversal by simultaneous coherent rotation ${ }^{5}$ for the increasing branch only. Improvement in the fits are achieved considering an intermixed layer only at the Co on Au interface. This asymmetric reversal is as expected following the asymmetric hysteresis loop observed in the case of a bilayer [Fig. 1(a)] for the respective field branches. Thus the separate ML case can be seen as only a scaling of the number of AF-FM interfaces from a bilayer, as no sequential switching is observed.

We explain the above observations assuming a simple model where the relevant energy terms can be written as

$$
E=-H_{a} M_{F M} \cos (\delta-\theta)-J M_{F M} M_{A F} \cos \delta+k \sin ^{2} \delta,
$$

where $J$ is the interlayer exchange between FM and AF layers. Here, $\delta$ is the angle between the $M_{F M}$ and the easy axis. $M_{F M}$ and $M_{A F}$ (uncompensated spins) are the saturation magnetization of the FM and AF layer and $k$ is the uniaxial anisotropy of the FM. Here we consider $M_{A F}$ along the easy axis which is assumed to be parallel to the $H_{F C}$ direction and $M_{A F}$ do not rotate with $H_{a}$ direction. ${ }^{14}$ For a finite $\theta$, the strength of the anisotropy field $H_{A}$ (dependent on the projec-

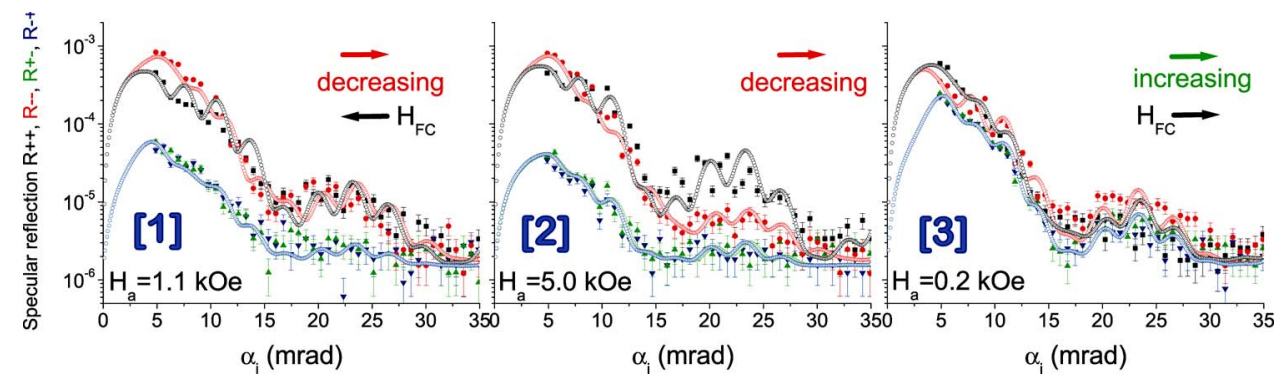

FIG. 4. (Color online) Measured (closed symbols) and fitted (open circle) NSF $\left(R_{++}\right.$and $\left.R_{--}\right)$and SF $\left(R_{+-}\right.$and $\left.R_{-+}\right)$reflectivity patterns of separate: $\mathrm{SiO}_{2} /[\mathrm{Co}(15.0 \mathrm{~nm}) / \mathrm{CoO}(8.0 \mathrm{~nm}) / \mathrm{Au}(46.0 \mathrm{~nm})]_{\times 20} \mathrm{ML}$ for increasing/decreasing applied fields $H_{a}$ where $H_{F C}=+4.0 /$ $-4.0 \mathrm{kOe}$, respectively. 


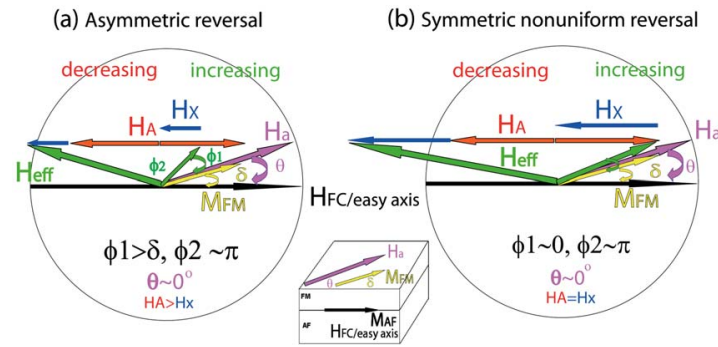

FIG. 5. (Color online) Sketch showing $H_{\text {eff }}$ (green) for $\theta \approx 0^{\circ}$ when (a) $H_{A}$ (red) $\gg H_{X}$ (blue) and (b) $H_{X} \geqslant H_{A}$. The $M_{F M}$ (yellow) and $H_{a}$ (violet) are making an angle $\delta$ and $\theta$, respectively, with the easy axis which is the $H_{F C}$ direction. The components of the fields $\perp$ to $H_{F C}$ has been exaggerated.

tion of the FM magnetization onto the easy axis) and that of the exchange field of the AF: $H_{X}\left(=J M_{A F}\right)$ decide on the angle between the effective field $H_{\text {eff }}\left(=H_{A}+H_{a}+H_{X}\right)$ and the $M_{F M}$ direction $\left(\phi_{1}\right)$ at equilibrium. As the sign of FM magnetization reverses so does the anisotropy field and the corresponding angle $\phi_{2}$ can be very close to $180^{\circ}$. Larger angle means larger torque, which favors rotation of the magnetization, whereas a small angle favors flipping by domain wall motion.

A sketch showing the angle which $H_{\text {eff }}$ makes with the $M_{F M}$ for representative strengths of the anisotropy and exchange field is presented in Fig. 5. When $H_{A}>H_{X}$ [Fig. 5(a)] a large angle $\phi_{1}$ is made for the increasing branch favoring rotation as $\phi_{1}>\delta$ while for the decreasing branch the angle $\phi_{2}$ can be as low as $\approx 180^{\circ}$. Our ML-S sample is in agreement with the above and shows asymmetric reversal. For such low values of $\theta$, one thus expects an asymmetric reversal in the case of a bilayer.

This is, however, not the case for the continuous ML (ML-C). From the fits to the neutron reflectivity patterns at various field strengths along the hysteresis loop, the sequen- tial layer-by-layer flipping revealed that each AF-FM interface down the stack are exchange coupled with increasing $H_{X}$ values. The effective angles $\phi_{1,2}$ therefore always remain very small, even though the $H_{A}$ values can be considered similar for ML-S and ML-C. Thus for $H_{X} \geqslant H_{A}$ [Fig. 5(b)] the angles $\phi_{1,2}$ for the increasing $\left(\simeq 0^{\circ}\right)$ as well as for the decreasing $\left(\simeq 180^{\circ}\right)$ field branches result in symmetric magnetization reversal which is only by flipping of the magnetization in absence of enough torque on the system. We believe, therefore, that the relative strengths of the $H_{X}$ and $H_{A}$ and thereby the effective field $H_{\text {eff }}$ and the angle it makes with the FM magnetization is responsible for the observed symmetric and asymmetric reversal in exchange bias systems. ${ }^{15}$ Our recent detailed study on the reversal for various $\theta$ has been seen to demonstrate the reversal process ${ }^{16}$ based upon the above arguments. The present observation is also in agreement with our previous observation on the $\mathrm{IrMn} / \mathrm{CoFe}$ system $^{8}$ with similar continuous AF-FM interfaces, thus we suggest that such symmetric reversal via domain structure formation is a general phenomena for such cases of continuous MLs.

In conclusion, we have studied the remagnetization behavior of the AF-FM interface in CoO-Co exchange-biased MLs for a continuous ML and compare it with that of a separated ML. For the ML-C the reversal of each FM layer proceeds sequentially and symmetrically via a nonuniform mode for both remagnetization directions, whereas the ML-S show usual asymmetric reversal. The behavior is understood in terms of increased exchange field strengths from successive interfaces down the stack in the case of a continuous multilayer compared to that of a separated multilayer or a bilayer with similar AF-FM interfaces. In accordance with the theoretical arguments, ${ }^{7}$ our experimental investigation shows in general the different reversal mechanisms for field applied along the field cooling direction and its dependence on the relative strengths of the Zeeman, exchange, and anisotropy energies in the system.
*Author to whom correspondence should be addressed. Electronic address: a.paul@fz-juelich.de

${ }^{1}$ W. H. Meiklejohn and C. P. Bean, Phys. Rev. 102, 1413 (1956).

${ }^{2}$ V. I. Nikitenko, V. S. Gornakov, A. J. Shapiro, R. D. Shull, Kai Liu, S. M. Zhou, and C. L. Chien, Phys. Rev. Lett. 84, 765 (2000).

${ }^{3}$ W.-T. Lee, S. G. E. te Velthuis, G. P. Felcher, F. Klose, T. Gredig, and E. D. Dahlberg, Phys. Rev. B 65, 224417 (2002).

${ }^{4}$ M. R. Fitzsimmons, P. Yashar, C. Leighton, Ivan K. Schuller, J. Nogues, C. F. Majkrzak, and J. A. Dura, Phys. Rev. Lett. 84, 3986 (2000).

${ }^{5}$ M. Gierlings, M. J. Prandolini, H. Fritzsche, M. Gruyters, and D. Riegel, Phys. Rev. B 65, 092407 (2002).

${ }^{6}$ F. Radu, M. Etzkorn, R. Siebrecht, T. Schmitte, K. Westerholt, and H. Zabel, Phys. Rev. B 67, 134409 (2003).

${ }^{7}$ B. Beckmann, U. Nowak, and K. D. Usadel, Phys. Rev. Lett. 91, 187201 (2003).

${ }^{8}$ A. Paul, E. Kentzinger, U. Rücker, D. E. Bürgler, and P. Grünberg, Phys. Rev. B 70, 224410 (2004).
${ }^{9}$ A. Paul, D. E. Bürgler, M. Luysberg, and P. Grünberg, Europhys. Lett. 70, 224410 (2004).

${ }^{10}$ L. G. Parratt, Phys. Rev. 95, 359 (1954).

${ }^{11}$ For information on HADAS see: http://www.fz-juelich.de/iff/ wns_hadas.

${ }^{12}$ H. Zabel, Appl. Phys. A: Solids Surf. 58, 259 (1994); E. Kentzinger, U. Rücker, and B. P. Toperverg, Physica B 335, 82 (2003).

${ }^{13}$ A. Paul, E. Kentzinger, U. Rücker, and Th. Brückel (unpublished).

${ }^{14}$ H. Ohldag, A. Scholl, F. Nolting, E. Arenholz, S. Maat, A. T. Young, M. Carey, and J. Stohr, Phys. Rev. Lett. 91, 017203 (2003).

${ }^{15}$ J. Camarero, J. Sort, A. Hoffmann, J. M. Garcia-Martin, B. Dieny, R. Miranda, and J. Nogues, Phys. Rev. Lett. 95, 057204 (2005).

${ }^{16}$ A. Paul, E. Kentzinger, U. Rücker, and Th. Brückel (unpublished). 